\title{
Baicalin promotes extracellular matrix synthesis in chondrocytes via the activation of hypoxia-inducible factor-1 $\alpha$
}

\author{
PENGZHEN WANG ${ }^{1}$, PINGPING ZHU ${ }^{2}$, RUIJIA LIU $^{3}$, QINGQI MENG ${ }^{3}$ and SIMING LI ${ }^{1,3}$ \\ ${ }^{1}$ Guangzhou Institute of Traumatic Surgery; Departments of ${ }^{2}$ Internal Neurology and ${ }^{3}$ Orthopedics, \\ Guangzhou Red Cross Hospital, Medical College, Jinan University, Guangzhou, Guangdong 510220, P.R. China
}

Received August 28, 2019; Accepted July 23, 2020

DOI: $10.3892 /$ etm.2020.9356

\begin{abstract}
Chinese herbal extracts are being used increasingly to treat osteoarthritis (OA) in recent years. Baicalin (BA) is an active component of Scutellaria baicalensis Georgi extracts and protects chondrocytes against damage. The aim of the present study was to examine the mechanism of action of BA on chondrocytes from mouse articular cartilage. In total, $44 \mu \mathrm{M}$ BA and $10 \mu \mathrm{M}$ hypoxia-inducible-factor- $1 \alpha$ (HIF-1 $\alpha$ ) inhibitor BAY-87-2243 were screened by the [3-(4,5-dimethylthiazol-2-yl)-5-(3-carboxymethoxyphenyl)-2(4-sulfophenyl)-2H-tetrazolium] method. Alcian blue and Safran $\mathrm{O}$ staining were used to investigate the synthesis of extracellular matrix (ECM) in chondrocytes treated with BA. The expression of HIF-1 $\alpha$ and chondrogenic marker genes including $S O X 9, A G G$ and $\operatorname{Col} 2 \alpha$ was detected by western blotting or reverse-transcription quantitative (RT-qPCR), the expression of $P H D 1,2,3$ and catabolic genes including ADAMTS5, MMP9 and MMP13 were detected by RT-qPCR. To investigate the effect of BA on the ECM synthesis of chondrocytes, $44 \mu \mathrm{M}$ BA and $10 \mu \mathrm{M}$ BAY were chosen for further experimentation. It was confirmed that BA at a concentration of $44 \mu \mathrm{M}$ could significantly promote the secretion of ECM. The expressions of genes including HIF-1 $\alpha$, SOX9, collagen type $2(\mathrm{Col} 2 \alpha)$ and aggrecan $(A G G)$ were elevated following BA pretreatment and decreased by subsequent BAY-87-2243 stimulation for $24 \mathrm{~h}$. Compared with untreated chondrocytes, the expressions of genes including ADAMTS5, MMP9, MMP13, PHD1, PHD2 and PHD3 in chondrocytes treated by BA were downregulated, however, BAY-87-2243 reversed the effect of BA on the genes including ADAMTS5, MMP9, MMP13, PHD1, PHD2 and PHD3 in chondrocytes. The
\end{abstract}

Correspondence to: Dr Siming Li or Dr Qingqi Meng, Department of Orthopedics, Guangzhou Red Cross Hospital, Medical College, Jinan University, 396 Tongfu Zhong Road, Guangzhou, Guangdong 510220, P.R. China

E-mail: drsmli@hotmail.com

E-mail: meng_qingqi@126.com

Key words: baicalin, hypoxia inducible factor-1 $\alpha$, SOX9, extracellular matrix, collagen type 2, aggrecan findings of the present study suggest that BA may promote ECM synthesis and marker gene expression in chondrocytes by activating HIF-1 $\alpha$. Therefore, BA may represent a novel clinical drug for OA.

\section{Introduction}

Osteoarthritis (OA) is the main cause of mobility-related disability caused by cartilage degeneration. Due to the rising average age of the global population in the United States, the prevalence of OA is expected to double by $2030(1,2)$. OA could lead to a decreased quality of life, the occurrence and formation of OA is because that articular cartilage has limited regenerative capacity due to poor cell migratory ability and low density (3). Once damaged, articular cartilage has a limited potential to repair $(4,5)$. The expression levels of matrix metalloproteinases (MMPs), including MMP9 and MMP13, in damaged articular chondrocytes are increased $(6,7)$. The extracellular matrix (ECM) of articular cartilage contains collagen type $2(\mathrm{Col} 2 \alpha)$ and aggrecan (AGG) synthesized by chondrocytes (8). Imbalances in ECM function lead to degenerative diseases, such as OA and cartilage injury (9), chondrocytes are stimulated to release MMPs, including MMP9, MMP13 and a disintegrin and metalloproteinase with thrombospondin motifs-5 (ADAMTS5) (10). It is well known that $A D A M T S 5$ and MMPs serve essential roles in degradation of cartilage ECM during the progression of OA (11).

Chondrogenesis is affected by a variety of factors, including growth factors such as TGF- $\beta$ (12) and BMP4 (13) and oxygen levels $(14,15)$. HIF-1 $\alpha$ in chondrocytes responds to oxygen concentration during cartilage differentiation and formation in a hypoxic microenvironment (16). When the oxygen concentration is close to normal, specific proline residues of HIF-1 $\alpha$ are rapidly hydroxylated and degraded by a series of proteasomes, including prolyl hydroxylase and asparaginyl hydroxylase (17). When the oxygen concentration is lower than the normal level, HIF-1 $\alpha$ is stable and binds to its counterpart HIF-1 $\alpha$ to form a heterodimer and relevant genes related to glucose metabolism, collagen production, angiogenesis and differentiation are activated and expressed $(18,19)$. For chondrocytes, the expression of SOX9, Col $2 \alpha$ and AGG can be activated and upregulated by stabilization and accumulation of $H I F-1 \alpha(16)$. 
Baicalin (BA) is a flavonoid compound extracted from the roots and stems of Scutellaria baicalensis Georgi and has a number of therapeutic effects, including antibacterial, diuretic, anti-inflammatory, anti-metamorphosis and antispasmodic effects, similar to those of other natural Chinese medicines, such as icariin and Salvianolic acid $(20,21)$. According to a report by Chen et al (21), flavonoids including BA are effective in the treatment of OA. Another study demonstrated that BA may maintain the phenotype of chondrocytes isolated from New Zealand rabbits (22). Previous studies have also demonstrated that BA inhibits the expression of inflammatory factors in articular chondrocytes by blocking the $N F-\kappa B$ pathway (23). It has also been confirmed that Bone Gla Protein (BGP, osteocalcin) promotes cartilage formation and glucose metabolism by activating HIF-1 $\alpha$ (24). Xing et al (23) concluded that BA protects chondrocytes by inhibiting $\mathrm{NF}-\kappa \mathrm{B}$ signaling pathways (23). Moreover, it was reported that BA significantly inhibited the oxidative stress and decreased cell apoptosis (25). The HIF-1 $\alpha$ pathway generally involves chondrogenesis and cartilage formation of chondrocytes (16). BAY-87-2243 is a potent and selective HIF-1 $\alpha$ blocker and inhibitor $(26,27)$, it is suggested that BAY-87-2243 could block of HIF- $1 \alpha$ expression mediated IL-17 and $\mathrm{CoCl}_{2}$ in RAW 264.7 macrophage cells (27). Although it is known that BA has a protective effect on chondrocytes, the effect and mechanism of BA on the HIF-1 $\alpha$ pathway in chondrocytes has not yet been elucidated.

Hence, the present study aimed to investigate the expression of chondrogenic genes and ECM in chondrocytes treated with or without BAY-87-2243 in the presence of BA. The regulatory mechanism of BA involving the HIF-1 $\alpha /$ SOX9 pathway was elucidated using molecularbiology experiments and morphological analysis. The present study provides a theoretical basis for clinical applications of BA to treat patients with cartilage damage and OA.

\section{Materials and methods}

Chemicals. BA was obtained from Bioruler Co., Ltd. Stock solutions of BA were dissolved in dimethyl sulfoxide (DMSO; Sigma-Aldrich; Merck KGaA) at room temperature and stored at $-20^{\circ} \mathrm{C}$. The final concentration of dimethyl sulfoxide used in the culture was $0.01 \%(\mathrm{v} / \mathrm{v})$, the concentration of BA working solution were $11,22,44,88$ and $176 \mu \mathrm{M}$. BAY-87-2243 was purchased from Selleck Chemicals. Stock solutions of BAY-87-2243 were dissolved in double-distilled water at room temperature and stored at $-20^{\circ} \mathrm{C}$, the working solution concentrations of BAY-87-2243 were 5, 10, 20 and $40 \mu \mathrm{M}$.

Animal experiments, cell culture and cytotoxicity analysis. Three to four weeks-old C57BL/6 male mice (13-20 g) were purchased from Medical Laboratory Animal Center (Guangzhou, China). The mice were bred and housed freely in a specific pathogen-free condition at a temperature of $18-22^{\circ} \mathrm{C}$ with a relative humidity of $50-60 \%$ on a $12 \mathrm{~h}$ light-dark cycle, with free access to water and food. The mice were kept for five days prior to chondrocytes isolation. Chondrocytes were isolated from the mice and digested according to a previously described protocol (28-30). Briefly, the mice $(n=30)$ were sacrificed using carbon dioxide gas $(28 \%$ chamber volume per min) under general anesthesia; chondrocytes were isolated immediately after the mice were euthanized. The knee cartilage was digested with collagenase I (Cat. no. C0130; Sigma-Aldrich; Merck KGaA) and collagenase D (cat. no. 11088858001, Roche Diagnostics) for $30 \mathrm{~min}$ at $37^{\circ} \mathrm{C}$. The isolated chondrocytes were seeded at a concentration of $5 \times 10^{5}$ cells $/ \mathrm{ml}$ onto a 10-cm diameter Petri dishes containing complete Dulbecco's Modified Eagle's Medium (DMEM; Gibco; Thermo Fisher Scientific, Inc.) containing $10 \%$ (v/v) fetal bovine serum (FBS; Gibco; Thermo Fisher Scientific, Inc.), $2 \mathrm{mM} / 1$ glutamine, $100 \mathrm{U} / \mathrm{ml}$ penicillin and $100 \mu \mathrm{g} / \mathrm{ml}$ streptomycin in a humidified atmosphere with $5 \% \mathrm{CO}_{2}$ at $37^{\circ} \mathrm{C}$, after three days of culture, the cell confluence reached 80 90\% and the harvested chondrocytes were used for subsequent experiments. For the [3-(4,5-dimethylthiazol-2-yl)-5-(3-carboxymethoxyphenyl)-2(4-sulfophenyl)-2H-tetrazolium] (MTS) assay, $\sim 4 \times 10^{3}$ chondrocytes were transferred to 96 -well plates to make final volumes of $0.2 \mathrm{ml} /$ well. For cytotoxicity analysis of BA, the medium was removed and the new medium containing gradient concentrations of BA $(11,22,44,88$ and $176 \mu \mathrm{M})$ was added on the next day, the new medium of the control groups contained no BA, the chondrocytes were treated with different concentrations of BA respectively for 12,24 and $48 \mathrm{~h}$. For cytotoxicity analysis of BAY-87-2243, the medium was removed and the new medium containing gradient concentrations of BAY-87-2243 $(5,10,20$ and $40 \mu \mathrm{M})$ was added on the next day, the new medium of control groups contained no BAY-87-2243, the chondrocytes were treated with different concentrations of BA respectively for 8,12 and $24 \mathrm{~h}$. The chondrocytes in all groups were incubated at $37^{\circ} \mathrm{C}$ in a $5 \% \mathrm{CO}_{2}$ container. Absorbance was determined at $490 \mathrm{~nm}$.

Alcian blue and Safranin O (SO) staining of cultured cells. Chondrocytes were seeded in a 12 -well plate at a density of $1 \times 10^{5}$ cells per well in a final volume of $10 \mu \mathrm{l}$. After $4 \mathrm{~h}$ of incubation for adherence of chondrocytes, $2 \mathrm{ml}$ of complete DMEM medium containing various concentrations of BA $(0,11,22,44$ and $176 \mu \mathrm{M})$ was added. After 14 days, cell masses were washed and fixed for $20 \mathrm{~min}$ with $0.5 \mathrm{ml}$ of $4.0 \%$ paraformaldehyde at room temperature and then stained with $0.1 \%$ (w/v) Safranin O (Sigma-Aldrich; Merck KGaA) for $30 \mathrm{~min}$ at room temperature and $1 \%(\mathrm{w} / \mathrm{v})$ Alcian blue (Sigma-Aldrich; Merck KGaA) for $1 \mathrm{~h}$ at room temperature. Photographs of stained cells were captured using an optical microscope (magnification, x4) (Microphot; Nikon Corporation) and analyzed using Image-Pro Plus 6.0 software (Media Cybernetics, Inc.).

Immunofluorescence. Chondrocytes at a concentration of $4 \times 10^{4} /$ well were seeded on round glass coverslips placed in 6 -well plates and chondrocytes were exposed to $44 \mu \mathrm{M}$ BA for $2 \mathrm{~h}$ followed by $10 \mu \mathrm{M}$ BAY-87-2243 for $24 \mathrm{~h}$. The cells on the coverslips were fixed for $15 \mathrm{~min}$ with $2 \mathrm{ml}$ of $4.0 \%$ paraformaldehyde at room temperature, the sections were blocked with $5 \%(\mathrm{v} / \mathrm{v})$ bovine serum albumin (BSA) for $1 \mathrm{~h}$ at room temperature and incubated with the primary antibody against HIF- $1 \alpha$ (1:50; cat. no. 36169; Cell Signaling Technology, Inc.) at $4^{\circ} \mathrm{C}$ overnight. Subsequently, the secondary fluorescein-conjugated goat anti-rabbit antibody (1:200; cat. no. ZF0311; OriGene 
Technologies, Inc.) was added and incubated for $1 \mathrm{~h}$ at room temperature and the cover slips were sealed. Images of stained cells were viewed and captured using confocal laser scanning microscopy (CLSM; Zeiss LSM 510 META System; magnification, $\mathrm{x} 400$ ) and compared to untreated cells.

Western blotting. Chondrocytes were seeded at a concentration of $2 \times 10^{5} /$ well in 6 -well plates, the chondrocytes were pretreated with BA at $44 \mu \mathrm{M}$ for $2 \mathrm{~h}$ and the chondrocytes were then treated or not treated with $10 \mu \mathrm{M}$ BAY-87-2243 for $24 \mathrm{~h}$ in the presence of BA. The chondrocytes in the all groups were incubated at $37^{\circ} \mathrm{C}$ in an incubator with $5 \%$ $\mathrm{CO}_{2}$. The cells were harvested using radioimmunoprecipitation assay buffer (cat. no. P0013D, Beyotime Institute of Biotechnology). Subsequently, $100 \mu$ l cell-lysate supernatants containing $1 \mathrm{mM}$ PMSF (Sigma-Aldrich; Merck KGaA) were analyzed using the bicinchoninic acid (BCA) protein quantitation kit. In total, $30 \mu \mathrm{g}$ of protein from each sample were separated on a $10 \%$ SDS-PAGE gel. The separated proteins were transferred onto polyvinylidene difluoride membranes (Bio-Rad Laboratories, Inc.). The membranes were soaked overnight at $4^{\circ} \mathrm{C}$ with solutions of primary rabbit anti-GAPDH antibody (1:1,000; cat. no. 5174), rabbit anti-HIF-1 $\alpha(1: 1,000$; cat. no. 36169), and rabbit anti-SOX9 antibodies $(1: 1,000$; cat. no. 82630; all Cell Signaling Technology, Inc.). The next day, the membranes were washed 3 times with PBS containing $0.1 \%$ Tween-20 (TBS-T). Diluted HRP-labeled goat anti-rabbit IgG (1:3,000; cat. no. ARG 65351; Arigo Biolaboratories) was added and left for $1 \mathrm{~h}$ at room temperature. GAPDH was used as the loading control. Images of the stained-protein bands were recorded using an ECL Western Blotting Substrate (Thermo Fisher Scientific, Inc.) kit and quantified using Image lab system version 2.0. (Bio-Rad Laboratories, Inc.).

Reverse-transcription quantitative $P C R(R T-q P C R)$. In total, $\sim 2 \times 10^{5}$ chondrocytes were transferred to 6-well plates and cultured at $37^{\circ} \mathrm{C}$ with $5 \% \mathrm{CO}_{2}$. The chondrocytes in control and treatments were pretreated with BA at $44 \mu \mathrm{M}$ for $2 \mathrm{~h}$ and the chondrocytes were then stimulated or not stimulated with $10 \mu \mathrm{M}$ BAY-87-2243 for $24 \mathrm{~h}$ in the presence of BA. Total RNA (Ribonucleic Acid) was extracted from chondrocytes using TRIzol reagent (Thermo Fisher Scientific, Inc.) and converted to cDNA using PrimeScript RT Master Mix (Takara Bio, Inc.). RT-qPCR was performed with SYBR Premix ExTaq (Takara Bio, Inc.) using the qTOWER version 3.0 PCR system (Jena Industries, Inc.). The thermocycling conditions were as follows: At $95^{\circ} \mathrm{C}$ for 2 min for pre-denaturation; 40 cycles of denaturation at $95^{\circ} \mathrm{C}$ for $34 \mathrm{sec}$, and annealing and extension at $55^{\circ} \mathrm{C}$ for $5 \mathrm{sec}$. The forward and reverse primers for all the target genes are presented in Table I. The expression of all genes was calculated by the $2^{-\Delta \Delta \mathrm{Cq}}$ method using $\beta$-actin as the control (29).

Statistical analysis. Results from 3 experimental repeats are presented as the mean \pm standard deviation. Comparisons were analyzed using analysis of variance (one-way ANOVA) followed by the post hoc Fisher's least significant difference or Bonferroni's tests using SPSS version 22 software (IBM Corp.). ${ }^{*} \mathrm{P}<0.05$ was considered to indicate a significant difference and ${ }^{* *} \mathrm{P}<0.01$ was considered to indicate a highly significant difference.

\section{Results}

Potential cytotoxicity of BA and BAY-87-2243 on chondrocytes was evaluated by MTS. BA is the most abundant and active component in Scutellaria baicalensis Georgi, BA has a chemical formula $\mathrm{C}_{21} \mathrm{H}_{18} \mathrm{O}_{11}$ (Fig. 1A) with molecular mass $446.361 \mathrm{~g} / \mathrm{mol}$ (30). The effect of BA on chondrocytes was firstly determined by the MTS method. Chondrocytes were subjected to the indicated BA concentration of $0,11,22,44,88$, and $176 \mu \mathrm{M}$ for 12, 24 and $48 \mathrm{~h}$. As demonstrated in Fig. 1B, BA at 11 and $22 \mu \mathrm{M}$ had no cytotoxicity on chondrocytes; BA at 44 and $88 \mu \mathrm{M}$ exhibited little cytotoxicity on chondrocytes and BA at $176 \mu \mathrm{M}$ exerted a significant cytotoxicity effect on chondrocytes. This trend of the effect of BA on chondrocytes remained almost consistent at 12, 24 and $48 \mathrm{~h}$ (Fig. 1B). BAY-87-2243, a potent and selective HIF-1 $\alpha$ inhibitor $(26,27)$, BAY-87-2243 at higher concentrations (10, 20 and $40 \mu \mathrm{M})$ had no effect on chondrocytes at the aforementioned 3 time points; however, BAY-87-2243 at a concentration of $10 \mu \mathrm{M}$ had no cytotoxicity on chondrocytes cultured for 8,6 and $24 \mathrm{~h}$ (Fig. 1C). The present study was designed to investigate the effect of BA on the ECM synthesis of chondrocytes instead of cell proliferation, hence, $44 \mu \mathrm{M}$ BA and $10 \mu \mathrm{M}$ BAY-87-2243 were chosen for further experimentation.

$B A$ promotes the expression of $S O X 9, A G G$ and Col $2 \alpha$ and ECM synthesis. SO and Alcian blue staining are classic methods for detecting the ECM component AGG (16), SO and Alcian blue staining indicated that BA (at 22, 44 and $88 \mu \mathrm{M}$ ) promoted the secretion of AGG in chondrocytes at varying degrees. Compared with untreated chondrocytes, AGG secretion from chondrocytes in BA $(44 \mu \mathrm{M})$ treated groups was significantly increased (Fig. 2A). In the present study, the protein level of SOX9 was significantly increased in the BA treatment groups compared with that of the control group $(\mathrm{P}<0.01)$ and the protein level of SOX9 was inhibited significantly by the addition of $10 \mu \mathrm{M}$ of BAY-87-2243 $(\mathrm{P}<0.01$; Fig. 2B and C). The mRNA expression of $S O X 9, A G G$ and Col $2 \alpha$ was significantly upregulated following BA treatment and the effect of BA on chondrocytes were obviously eliminated by BAY-87-2243 ( $\mathrm{P}<0.01$; Fig. 2D-F). These results indicated that ECM synthesis and chondrogenic expressionin chondrocytes were upregulated by BA and downregulated by BAY-87-2243.

$B A$ activates HIF-1 $\alpha$ expression in chondrocytes compared with the untreated chondrocytes. HIF-1 $\alpha$ (green) nuclei expression was increased in chondrocytes treated with BA for $24 \mathrm{~h}$, compared with the BA (only)-treated chondrocytes. HIF-1 $\alpha$ (green) nuclei expression was decreased significantly in chondrocytes treated by BAY-87-2243 in the presence of BA (Fig. 2A). Simultaneously, BA stimulated an upregulation in HIF-1 $\alpha$ mRNA $(\mathrm{P}<0.01$; Fig. 3B) and HIF-1 $\alpha$ protein expression $(\mathrm{P}<0.01$; Fig. $3 \mathrm{C}$ and $\mathrm{D})$ in the chondrocytes in accordance with the observed upregulation of HIF-1 $\alpha$ stabilization and nuclear translocation in immunofluorescence experiments. The expression level of HIF-1 $\alpha$ in chondrocytes 
Table I. Sequences of primers used for gene amplification by reverse-transcription quantitative PCR.

\begin{tabular}{lll}
\hline Genes & \multicolumn{1}{c}{ Forward primer sequence (5'-3') } & \multicolumn{1}{c}{ Reverse primer sequence (5'-3') } \\
\hline$\beta$-actin & ATTGTGCACCGCAAATGCTT & ACCACAGCACGATTGTCGAT \\
HIF-1 $\alpha$ & CCACCACAACTGCCACCACTG & TGCCACTGTATGCTGATGCCTTAG \\
SOX9 & TCAACGGCTCCAGCAAGAACAAG & CTCCGCCTCCTCCACGAAGG \\
Col2 $\alpha$ & GGTCCTCCTGGTCCTGGCATC & CGTGCTGTCTCAAGGTACTGTCTG \\
AGG & CGTTGCAGACCAGGAGCAAT & CTCGGTCATGAAAGTGGCGG \\
MMP9 & AGAGACCACCACCACCACCAC & TGCCTGCCTCCACTCCTTCC \\
MMP13 & CTACCATCCTGCGACTCTTGCG & CCACATCAGGCACTCCACATCTTG \\
ADAMTS5 & AAGAGGAGGAGGAGGAGGAGGAG & AATGGTTGTGAGCTGCCGTATGG \\
PHD1 & AGGCTATGTCCGTCACGTTG & TGGGCTTTGCCTTCTGGAAA \\
PHD2 3 ATATTGTGCCTTGCATGCGG & TGGCTCACTAGTTGCCCATC \\
\hline
\end{tabular}

HIF- $1 \alpha$, hypoxia-inducible factor- $1 \alpha$; AGG, aggrecan; Col2 $\alpha$, collagen type 2; MMP, matrix metalloproteinase; ADAMTS5, a disintegrin and metalloproteinase with thrombospondin motifs 5; PHD, prolyl hydroxylase.<smiles>O=C(O)C1OC(Oc2cc3oc(-c4ccccc4)cc(=O)c3c(O)c2O)[C@H](O)[C@H](O)[C@@H]1O</smiles>

B

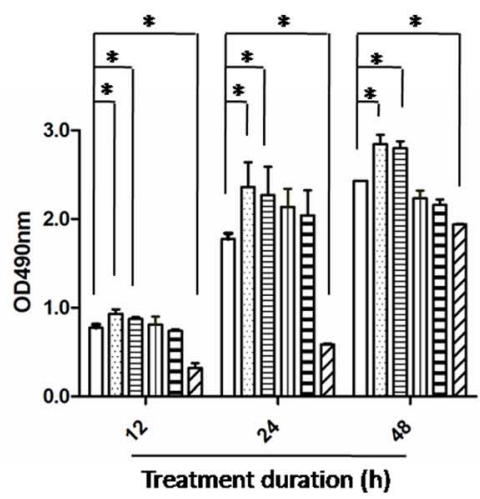

C
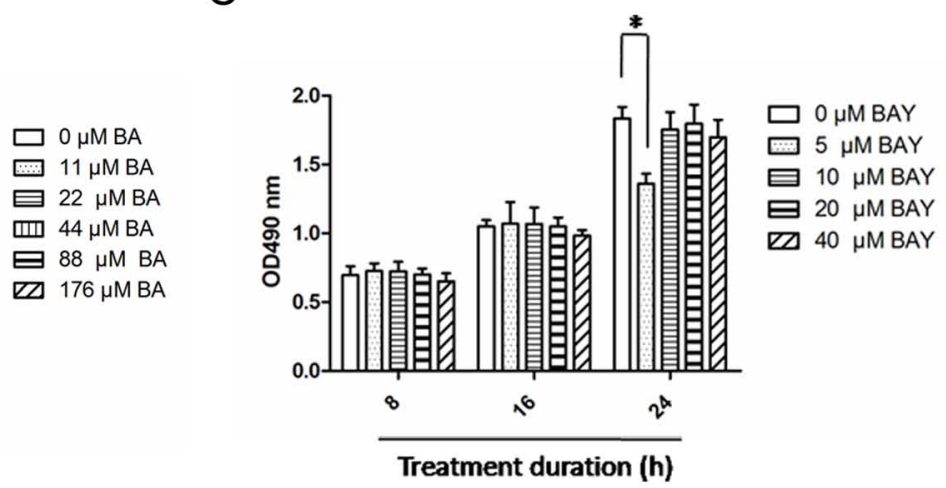

Figure 1. Effects of BA and BAY-87-2243 on chondrocytes. (A) Molecular structure of BA. (B) Chondrocytes were treated with BA (0, 11, 22, 44, 88 and $176 \mu \mathrm{M})$ for 12,24 and $48 \mathrm{~h}$ and cytotoxicity was assessed using the MTS assay. (C) Chondrocytes were treated for 8,16 and $24 \mathrm{~h}$ with BAY (5, 10 , 20 and $40 \mu \mathrm{M}$ ) and the cytotoxicity was determine by MTS assay. "P<0.05. BA, baicalin; BAY, hypoxia inducible factor-1 $\alpha$ inhibitor (BAY-87-2243); MTS, [3-(4,5-dimethylthiazol-2-yl)-5-(3-carboxymethoxyphenyl)-2-(4-sulfophenyl)-2H-tetrazolium], comparisons were analyzed using one-way ANOVA followed by Bonferroni's tests.

treated with BA was decreased significantly by BAY (Fig. 3). These results indicated that BA could activate the nuclear expression of HIF-1 $\alpha$.

BA downregulates MMP9, MMP13, ADAMTS5 and prolyl hydroxylases (PHD) genes expression. MMPs (especially MMP9 and MMP13) and ADAMTS5 are regarded as the key enzymes in the progression of OA that involves the breakdown of the ECM (31). Compared with respective control groups, the mRNA expression of ADAMTS5, MMP9, MMP13, PHD1, $P H D 2$ and $P H D 3$ in chondrocytes of BA-treated groups were significantly downregulated $(\mathrm{P}<0.01$; Fig. 4A-F). Compared with the BA-treated chondrocytes, the mRNA expression of ADAMTS5, MMP9, MMP13, PHD1, PHD2 and PHD3 were increased significantly in chondrocytes treated by BAY-87-2243 in the presence of BA (Fig. 4A-F). These results 
A

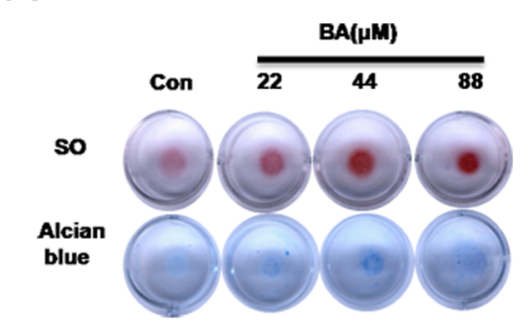

D

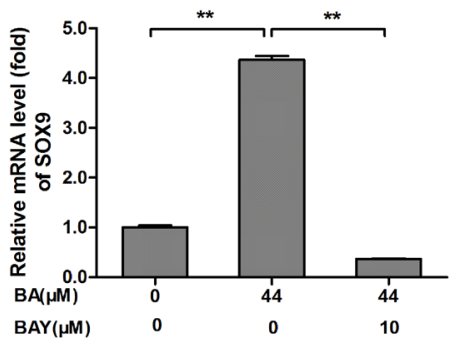

B

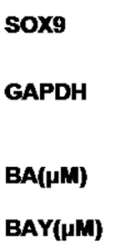

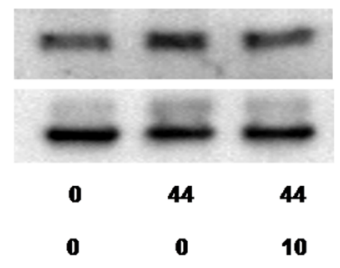

E

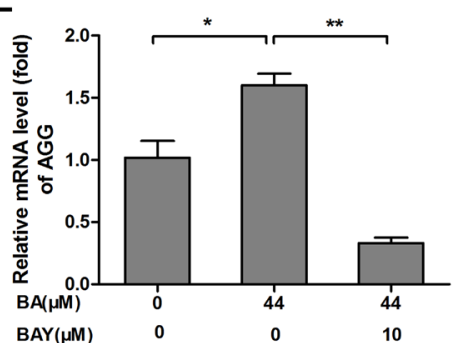

C

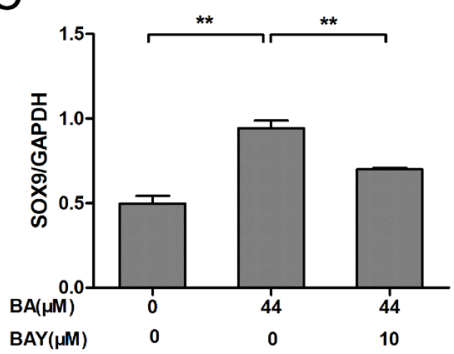

F

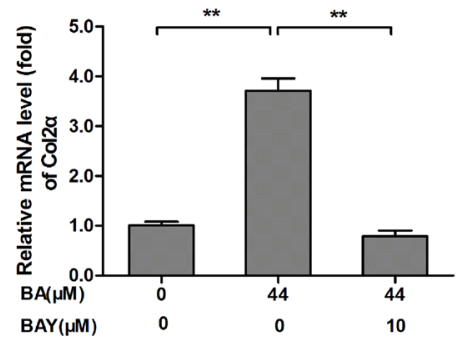

Figure 2. BA enhances extracellular matrix synthesis and chondrogenic marker expression, while the effect was decreased by the HIF-1 $\alpha$ inhibitor BAY(BAY-87-2243). (A) The chondrocyte masses treated by gradient concentration of BA $(0,22,44$ and $88 \mu \mathrm{M})$ were stained by SO and Alcian blue staining (4x). (B-D) Protein and mRNA expression of SOX9 was analyzed by western blotting and RT-qPCR, respectively and quantified using histograms). (E) AGG and (F) Col $2 \alpha$ mRNA expression was analyzed by RT-qPCR and $\beta$-actin was used as an internal control for RT-qPCR. ${ }^{*} \mathrm{P}<0.05$, ** $<<0.01$. HIF-1 $\alpha$, hypoxia-inducible factor-1 $\alpha$; BA, baicalin; BAY, hypoxia inducible factor-1 $\alpha$ inhibitor (BAY-87-2243); SO, Safranin O; RT-qPCR, reverse-transcription quantitative; AGG, aggrecan; Col2 $\alpha$, collagen type 2; con, control.

indicated that BA exerted a protective effect on chondrocytes by inhibiting the expression of catabolic genes.

\section{Discussion}

In the present study, it was found that BA promoted the synthesis of ECM in chondrocytes. In addition, it was found that the effect of BA on chondrocytes was dependent on the activation and expression of HIF-1 $\alpha$ by BA. The addition of the HIF-1 $\alpha$ inhibitor BAY-87-2243 significantly inhibited the expression and activation of HIF-1 $\alpha$. The findings of the present study indicated that BA may be a candidate drug to treat patients with cartilage damage and OA.

As shown in Fig. 1B, Low concentrations of BA (11 and $22 \mu \mathrm{M})$ had no cytotoxic effect on chondrocyte, however, high concentrations of BA $(176 \mu \mathrm{M})$ had significant cytotoxic effect on chondrocytes. Notably, it has been reported that BA at $1.25 \mu \mathrm{M}$ increased rabbit primary chondrocyte proliferation (32). The present study found that primary mouse chondrocytes were less sensitive to BA compared with rabbit chondrocytes used in the aforementioned study. It also indicated that low concentration BA $(11$ and $22 \mu \mathrm{M})$ promoted cell proliferation, suitable concentration of BA $(11$ and $22 \mu \mathrm{M})$ had no toxic side effects on chondrocytes, high concentration BA $(176 \mu \mathrm{M})$ had cytotoxic effect toxic side effects on chondrocytes. In the present study, the effect of BA on the ECM synthesis and the expression of HIF-1 $\alpha$ in chondrocytes was explored. In addition, $44 \mu \mathrm{M}$ BA was demonstrated to have no proliferative effect or side effects on chondrocytes and was chosen for subsequent experiments.

In the present research, $10 \mu \mathrm{M}$ BAY-87-2243 had no cytotoxicity effect on chondrocytes, which was similar with the the conclusion reported in literature (33). Therefore, $10 \mathrm{uM}$ BAY-87-2243 was used for western blotting, RT-qPCR and immunofluorescence experiments. In the present study, the effects of BA on chondrocyte ECM production and function were examined by $\mathrm{SO}$ and Alcian blue staining and it was demonstrated that BA promoted AGG synthesis in chondrocytes. The results of the present study demonstrated that BA treatment promoted AGG synthesis by chondrocytes at concentrations ranging from $22-88 \mu \mathrm{M}$. Specifically, BA at $44 \mu \mathrm{M}$ had the strongest effect on AGG synthesis and secretion. Further, the upregulation of $\mathrm{A} G G$ and Col $2 \alpha$ mRNA supported the conclusion that the BA exerted an effect on ECM synthesis. Key catabolic genes including ADAMTS5, MMP9, and MMP13 mRNA levels were downregulated following BA treatment. BAY-87-2243 significantly reversed the above effects of BA. The findings of the present study indicated that BA inhibited the expressions of catabolic genes including MMP9, MMP13, PHD1, PHD2 and PHD3.

In the present study, in order to explore the mechanism of action of BA on chondrocytes, HIF-1 $\alpha$ expression was examined after treatment with BA and HIF-1 $\alpha$ inhibitor BAY-87-2243. The expression of HIF-1 $\alpha$ and SOX9 was significantly increased as was the mRNA expression of AGG and $\mathrm{Col} 2 \alpha$ in chondrocytes compared to the control cells. However, BAY-87-2243 inhibited the expression of HIF-1 $\alpha$ and SOX9 in the present study. Previous studies have reported that HIF-1 $\alpha$ exhibited cartilage-formation ability and can also maintain chondrocyte phenotype by regulating SOX9 expression (34-36), Using a HIF-1 $\alpha$ deletion model, our previous study indicated that icariin (ICA) increased ECM expression by activating HIF-1 $\alpha$ (37), the conclusion of the present study was consistent with those studies reported previously. 
A

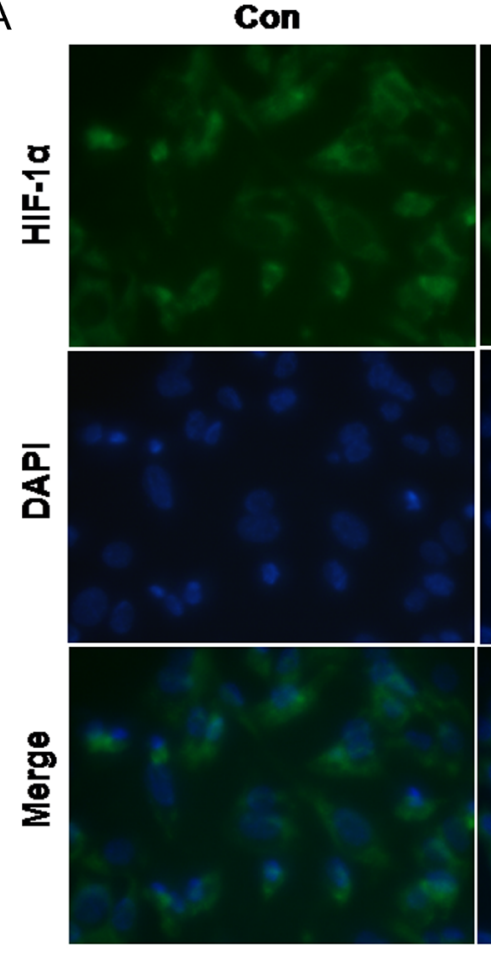

B

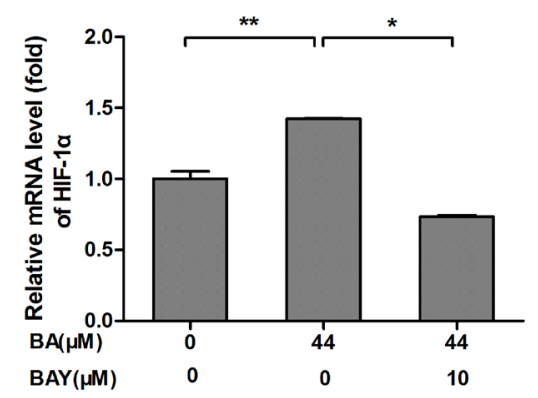

BA

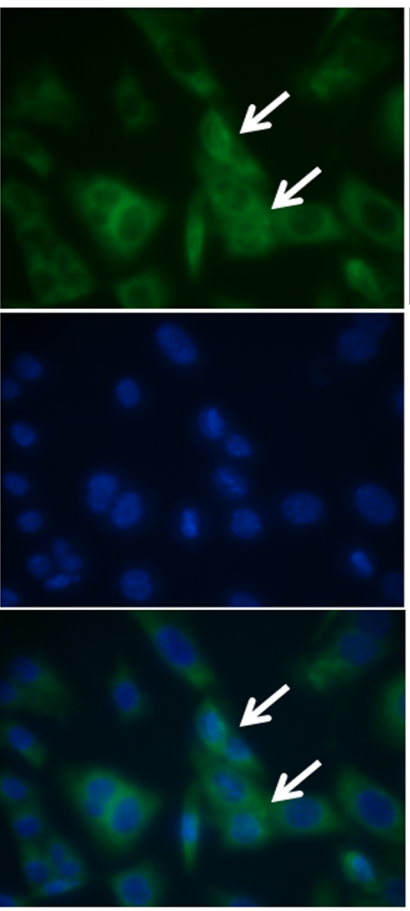

C

HIF-1a

GAPDH

BA(pM)

BAY( $\mu \mathrm{M})$

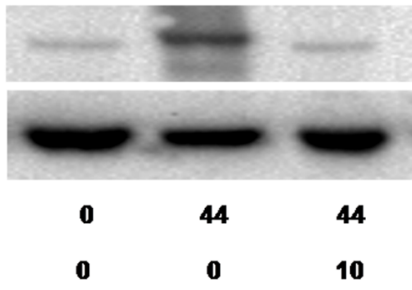

BA+BAY

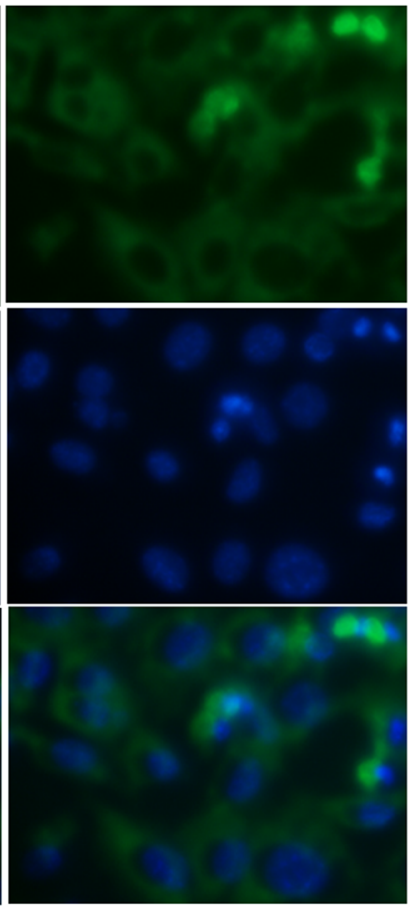

D

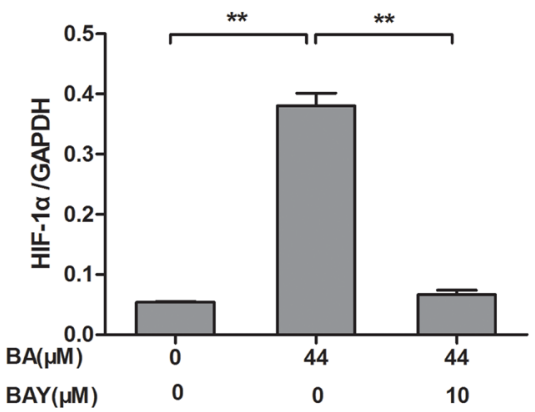

Figure 3. BA promotes the expression of HIF-1 $\alpha$ in newborn-mouse cartilage chondrocytes and this effect was reversed by BAY. (A) HIF-1 $\alpha$ nuclear localization in BA $(44 \mu \mathrm{M})$-treated chondrocytes with or without BAY $(10 \mu \mathrm{M})$ was observed under a fluorescence microscope (magnification, $400 \mathrm{x})$, the white arrows indicate chondrocytes with strong nuclear HIF-1 $\alpha$ expression. (B-D) mRNA and protein levels of HIF-1 $\alpha$ measured by RT-qPCR and western blotting, respectively. $\beta$-actin was the control for RT-qPCR and GAPDH was the control for western blotting. ${ }^{*} \mathrm{P}<0.05$, ${ }^{* *} \mathrm{P}<0.01$. HIF-1 $\alpha$, hypoxia-inducible factor-1 $\alpha$; BA, baicalin; BAY, hypoxia inducible factor-1 $\alpha$ inhibitor (BAY-87-2243); RT-qPCR, reverse-transcription quantitative; con, control; BAY, hypoxia inducible factor- $1 \alpha$ inhibitor.

In the present study, $P H D 1$, PHD2 and $P H D 3$ mRNA expression significantly decreased following BA treatment. HIF-1 $\alpha$ is usually induced by hypoxia, in addition, it has also been suggested that certain flavonoids sequester iron ions that are cofactors required for PHD activity, hence leading to the inactivation of PHDs (38). Once PHDs are inactivated, HIF-1 $\alpha$ enters the nucleus and then activates the expression of the downstream genes including $S O X 9$ and $A G G$ (39). It was documented that some flavonoids (such as quercetin, galangin and icariin) chelated cellular iron ions, a required cofactor for PHD activity, leading to the inhibition of PHD-catalyzed HIF prolyl hydroxylation and subsequently HIF- $1 \alpha / 2 \alpha$ accumulation (38-40). 
A

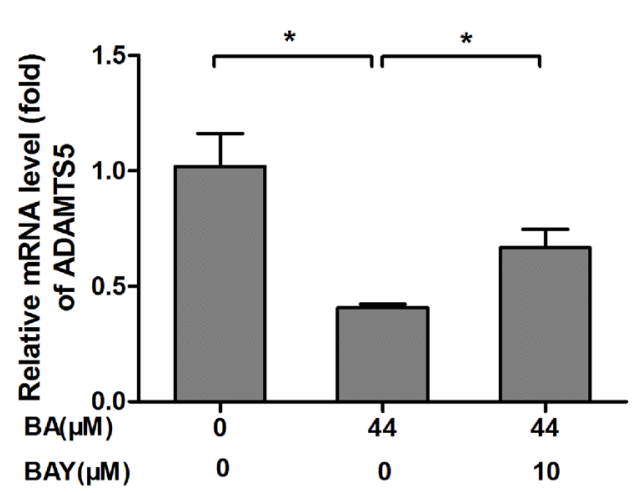

C

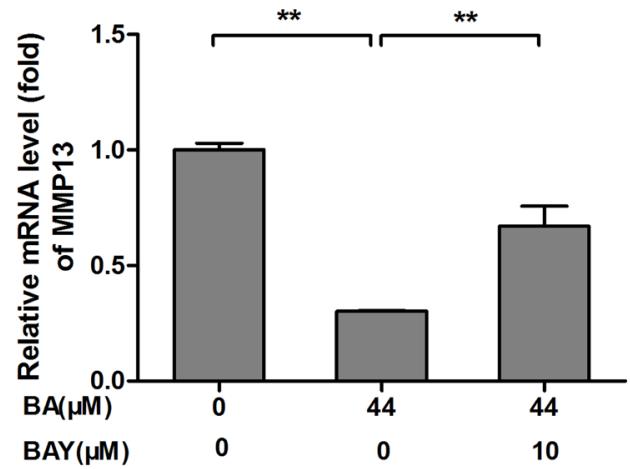

$E$

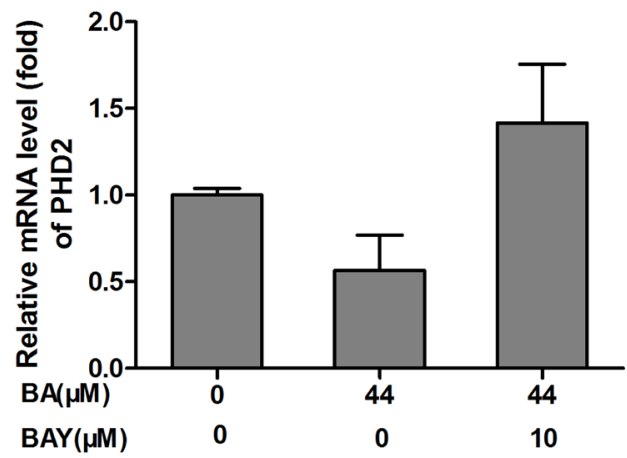

$\mathrm{B}$

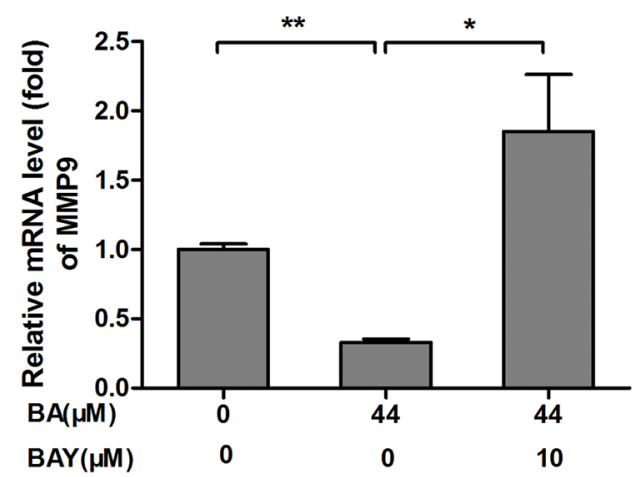

D

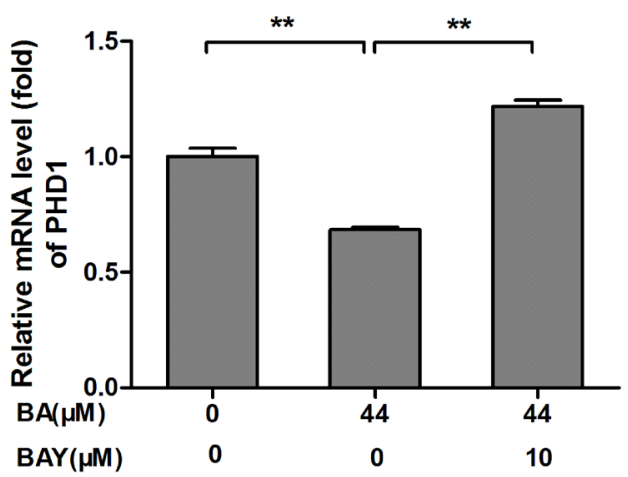

$\mathrm{F}$

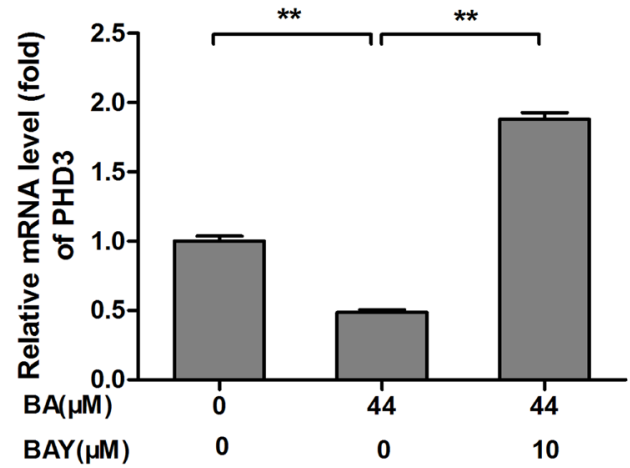

Figure 4. BA treatment decreased chondrogenic catabolic genes and PHD expression, the effect was blocked by the HIF-1 $\alpha$ inhibitor BAY. Chondrocytes originating from newborn mice were treated with or without $10 \mu \mathrm{M}$ BAY in the presence of BA (44 $\mu \mathrm{M})$ for $24 \mathrm{~h}$. (A) ADAMTS5, (B) MMP9 and (C) MMP13 mRNA expression was detected by RT-qPCR. (D) PHD1, (E) PHD2 and (F) PHD3 mRNA expression was detected by RT-qPCR, $\beta$-actin was the control for RT-qPCR. " $\mathrm{P}<0.05,{ }^{* *} \mathrm{P}<0.01$. HIF-1 $\alpha$, hypoxia-inducible factor-1 $\alpha$; BA, baicalin; BAY, hypoxia inducible factor-1 $\alpha$ inhibitor (BAY-87-2243); RT-qPCR, reverse-transcription quantitative; con, control; MMP, matrix metalloproteinase; ADAMTS5, a disintegrin and metalloproteinase with thrombospondin motifs 5; PHD, prolyl hydroxylase.

Thus, it was determined whether BA induced the accumulation of HIF-1 $\alpha$ in chondrocytes through the similar mechanism as other flavonoids. It was revealed that the protein expression of PHD1, PHD2 and PHD3 in chondrocytes was also reduced by BA treatment. It is purported that protein levels and activity of the hypoxia-inducible transcription factors HIF-1 $\alpha$ is controlled by hydroxylation of the regulatory $\alpha$ chains. Proline hydroxylases (PHDs) target the protein for degradation via the von-Hippel-Lindau-ubiquitin-ligase complex, and asparagine hydroxylation by Factor Inhibiting HIF leads to transcriptional inactivation (41-43). The present study only evaluated inhibition and accumulation of HIF-1 $\alpha$ in chondrocytes treated by BA; however, the method by which BA regulates PHD expression and whether the reduction in PHD expression also contributes to the HIF-1 $\alpha$ accumulation and has other biological significance in chondrocytes, need to be further studied.

The present study had some limitations. Firstly, the effectof BA on chondrocytes was not studied by performing chelation experiments. This can be the focus of research for further studies. Secondly, there was no in vivo experiments performed in the present study. Future studies are required for in vivo verification of the findings of the present study. Further studies are also required to investigatethe pharmacological mechanisms of BA effects on chondrocytes. 
In summary, it was confirmed that BA exerted a protective effect on mouse chondrocytes in vitro. In addition, it was concluded that BA promoted ECM synthesis at least partly by activating the HIF-1 $\alpha /$ SOX 9 pathway. The findings of the present study may lead to BA being developed as a potential drug to treat patients with $\mathrm{OA}$.

\section{Acknowledgements}

Not applicable.

\section{Funding}

This study was funded by grants from the Guangzhou Municipal Health and Family Planning Commission (grant nos. 20191A011018 and 20192A010009 to PW) and Guangzhou Science and Technology Project (grant nos. 201607010009 to SL and 201607010010 to QM).

\section{Availability of data and materials}

The datasets used and/or analyzed during the current study are available from the corresponding author on reasonable request.

\section{Authors' contributions}

SL, QM and PW conceived and designed the experiments; PW performed the majority of the experiments; QM and RL performed the MTS assay and parts of the western blotting experiments; PZ performed the immunofluorescence experiments and analyzed the data; SL, QM and PW analyzed data; SL, PW and QM wrote the manuscript. All authors have read and approved the manuscript.

\section{Ethics approval and consent to participate}

Animal experiments were approved (approval no. 2018-002-01) by the Ethics Committee of Guangzhou Red Cross Hospital (Guangzhou, China).

\section{Patient consent for publication}

Not applicable.

\section{Competing interests}

The authors declare that they have no competing interests.

\section{References}

1. Thomas AC, Hubbard-Turner T, Wikstrom EA and PalmieriSmith RM: Epidemiology of posttraumatic osteoarthritis. J Athl Train 52: 491-496, 2017.

2. Centers for Disease Control and Prevention (CDC): Public health and aging: projected prevalence of self-reported arthritis or chronic joint symptoms among persons aged $>65$ years--United States, 2005-2030. MMWR Morb Mortal Wkly Rep. 52(21):489491, 2003.

3. Zhou X, Tenaglio S, Esworthy T, Hann SY, Cui H, Webster TJ, Fenniri $\mathrm{H}$ and Zhang LG: Three-Dimensional printing biologically inspired DNA-based gradient scaffolds for cartilage tissue regeneration. ACS Appl Mater Interfaces 12: 33219-33228, 2020.
4. Frenkel SR, Clancy RM, Ricci JL, Di Cesare PE, Rediske JJ and Abramson SB: Effects of nitric oxide on chondrocyte migration E, adhesion, and cytoskeletal assembly. Arthritis Rheum 39: 1905-1912, 2015

5. Heinemeier KM, Schjerling P, Heinemeier J, Møller MB, Krogsgaard MR, Grum-Schwensen T, Petersen MM and Kjaer M: Radiocarbon dating reveals minimal collagen turnover in both healthy and osteoarthritic human cartilage. Sci Transl Med 8: 346ra90, 2016.

6. Oda K and Minata M: Drug free remission after steroid-dependent disappearance of lymphoproliferative disorder in rheumatoid arthritis patient treated with TNF-alpha blockade: Case study. Springerplus 4: 41, 2015.

7. Yammani RR, Carlson CS, Bresnick AR and Loeser RF: Increase in production of matrix metalloproteinase 13 by human articular chondrocytes due to stimulation with S100A4: Role of the receptor for advanced glycation end products. Arthritis Rheum 54: 2901-2911, 2006.

8. Thomas CM, Fuller CJ, Whittles CE and Sharif M: Chondrocyte death by apoptosis is associated with cartilage matrix degradation. Osteoarthritis Cartilage 15: 27-34, 2007.

9. Goldring MB, Fukuo K, Birkhead JR, Dudek E and Sandell LJ: Transcriptional suppression by interleukin-1 and interferon-gamma of type II collagen gene expression in human chondrocytes. J Cell Biochem 54: 85-99, 1994.

10. Zheng W, Tao Z, Chen C, Zhang C, Zhang H, Ying X and Chen $\mathrm{H}$ : Plumbagin prevents IL-1 $\beta$-induced inflammatory response in human osteoarthritis chondrocytes and prevents the progression of osteoarthritis in mice. Inflammation 40: 849-860, 2017.

11. Xue M, McKelvey K, Shen K, Minhas N, March L, Park SY and Jackson CJ: Endogenous MMP-9 and not MMP-2 promotes rheumatoid synovial fibroblast survival, inflammation and cartilage degradation. Rheumatology (Oxford) 53: 2270-2279, 2014.

12. Alvarez J, Horton J, Sohn P and Serra R: The perichondrium plays an important role in mediating the effects of TGF-beta1 on endochondral bone formation. Dev Dyn 221: 311-321, 2001.

13. Kim BS, Kang KS and Kang SK: Soluble factors from ASCs effectively direct control of chondrogenic fate. Cell Prolif 43: 249-261, 2010

14. Rodenas-Rochina J, Kelly DJ, Gómez Ribelles JL and Lebourg M: Influence of oxygen levels on chondrogenesis of porcine mesenchymal stem cells cultured in polycaprolactone scaffolds. J Biomed Mater Res A 105: 1684-1691, 2017.

15. Zhang FJ, Luo W and Lei GH: Role of HIF- $1 \alpha$ and HIF- $2 \alpha$ in osteoarthritis. Joint Bone Spine 82: 144-147, 2015.

16. Hu S, Zhang C, Ni L, Huang C, Chen D, Shi K, Jin H, Zhang K, Li Y, Xie L, et al: Stabilization of HIF-1 $\alpha$ alleviates osteoarthritis via enhancing mitophagy. Cell Death Dis 11: 481, 2020.

17. Hirota K and Semenza GL: Regulation of hypoxia-inducible factor 1 by prolyl and asparaginyl hydroxylases. Biochem Biophys Res Commun 338: 610-616, 2005.

18. Araldi E and Schipani E: Hypoxia, HIFs and bone development. Bone 47: 190-196, 2010.

19. Denko NC: Hypoxia, HIF1 and glucose metabolism in the solid tumour. Nat Rev Cancer 8: 705-713, 2008.

20. Chen H, Xu Y, Wang J, Zhao W and Ruan H: Baicalin ameliorates isoproterenolinduced myocardial infarction through iNOS, inflammation and oxidative stress in rat. Int J Clin Exp Pathol 8: 10139-10147, 2015.

21. Chen C, Zhang C, Cai L, Xie H, Hu W, Wang T, Lu D and Chen H: Baicalin suppresses IL-1 $\beta$-induced expression of inflammatory cytokines via blocking NF- $\kappa \mathrm{B}$ in human osteoarthritis chondrocytes and shows protective effect in mice osteoarthritis models. Int Immunopharmacol 52: 218-226, 2017.

22. Zhu M, Ying J, Lin C, Wang Y, Huang K, Zhou Y and Teng H: Baicalin induces apoptotic death of human chondrosarcoma cells through mitochondrial dysfunction and downregulation of the PI3K/Akt/mTOR pathway. Planta Med 85: 360-369, 2019.

23. Xing D, Gao H, Liu Z, Zhao Y and Gong M: Baicalin inhibits inflammatory responses to interleukin- $1 \beta$ stimulation in human chondrocytes. J Interferon Cytokine Res 37: 398-405, 2017.

24. Idelevich A, Rais Y and Monsonego-Ornan E: Bone Gla protein increases HIF-1alpha-dependent glucose metabolism and induces cartilage and vascular calcification. Arterioscler Thromb Vasc Biol 31: e55-e71, 2011.

25. Pan Y, Chen D, Lu Q, Liu L, Li X and Li Z: Baicalin prevents the apoptosis of endplate chondrocytes by inhibiting the oxidative stress induced by H2O2. Mol Med Rep 16: 2985-2991, 2017. 
26. Griggio V, Vitale C, Todaro M, Riganti C, Kopecka J, Salvetti C, Bomben R, Bo MD, Magliulo D, Rossi D, et al: HIF-1 $\alpha$ is over-expressed in leukemic cells from TP53-disrupted patients and is a promising therapeutic target in chronic lymphocytic leukemia. Haematologica 105: 1042-1054, 2020.

27. Samarpita S, Doss HM, Ganesan R and Rasool M: Interleukin 17 under hypoxia mimetic condition augments osteoclast mediated bone erosion and expression of HIF-1 $\alpha$ and MMP-9. Cell Immunol 332: 39-50, 2018.

28. Gosset M, Berenbaum F, Thirion S and Jacques C: Primary culture and phenotyping of murine chondrocytes. Nat Protoc 3 1253-1260, 2008.

29. Livak KJ and Schmittgen TD: Analysis of relative gene expression data using real-time quantitative PCR and the 2(-Delta Delta C(T)) method. Methods 25: 402-408, 2001.

30. Chen C, Zhang C, Cai L, Xie H, Hu W, Wang T, Lu D and Chen H: Baicalin suppresses IL-1 $\beta$-induced expression of inflammatory cytokines via blocking NF- $\mathrm{BB}$ in human osteoarthritis chondrocytes and shows protective effect in mice osteoarthritis models Int Immunopharmacol 52: 218-226, 2017.

31. He Y, Moqbel SAA, Xu L, Ran J, Ma C, Xu K, Bao J, Jiang L, Chen W, Xiong Y and Wu L: Costunolide inhibits matrix metalloproteinases expression and osteoarthritis via the NF- $\mathrm{KB}$ and Wnt $/ \beta$-catenin signaling pathways. Mol Med Rep 20: 312-322, 2019.

32. Huang $\mathrm{X}, \mathrm{Wu} \mathrm{H}$, Wang $\mathrm{L}$, Zheng $\mathrm{L}$ and Zhao J: Protective effects of baicalin on rabbit articular chondrocytes in vitro. Exp Ther Med 13: 1267-1274. 2017

33. Ellinghaus P, Heisler I, Unterschemmann K, Haerter M, Beck H, Greschat S, Ehrmann A, Summer H, Flamme I, Oehme F, et al: BAY 87-2243, a highly potent and selective inhibitor of hypoxia-induced gene activation has antitumor activities by inhibition of mitochondrial complex I. Cancer Med 2: 611-624, 2013.

34. Robins JC, Akeno N, Mukherjee A, Dalal RR, Aronow BJ, Koopman P and Clemens TL: Hypoxia induces chondrocyte-specific gene expression in mesenchymal cells in association with transcriptional activation of Sox9. Bone 37: 313-322, 2005.
35. Lefebvre V and Dvir-Ginzberg M: SOX9 and the many facets of its regulation in the chondrocyte lineage. Connect Tissue Res 58: 2-14, 2017.

36. Duval E, Baugé C, Andriamanalijaona R, Bénateau H, Leclercq S, Dutoit S, Poulain L, Galéra P and Boumédiene K: Molecular mechanism of hypoxia-induced chondrogenesis and its application in in vivo cartilage tissue engineering. Biomaterials 33: 6042-6051, 2012

37. Wang P, Zhang F, He Q, Wang J, Shiu HT, Shu Y, Tsang WP, Liang S, Zhao $\mathrm{K}$ and Wan C: Flavonoid compound icariin activates hypoxia inducible factor-1 $\alpha$ in chondrocytes and promotes articular cartilage repair. PLoS One 11: e0148372, 2016.

38. Park SS, Bae I and Lee YJ: Flavonoids-induced accumulation of hypoxia-inducible factor (HIF)-1alpha/2alpha is mediated through chelation of iron. J Cell Biochem 103: 1989-1998, 2008.

39. Leopoldini M, Russo N, Chiodo S and Toscano M: Iron chelation by the powerful antioxidant flavonoid quercetin. J Agric Food Chem 54: 6343-6351, 2006.

40. Davis CK, Jain SA, Bae ON, Majid A and Rajanikant GK: Hypoxia mimetic agents for ischemic stroke. Front Cell Dev Biol 6: 175, 2019.

41. Terkhorn SP, Bohensky J, Shapiro IM, Koyama E and Srinivas V: Expression of HIF prolyl hydroxylase isozymes in growth plate chondrocytes: Relationship between maturation and apoptotic sensitivity. J Cell Physiol 210: 257-265, 2007.

42. Wang W, Xu B, Xuan H, Ge Y, Wang Y, Wang L, Huang J, $\mathrm{Fu}$ W, Michie SA and Dalman RL: Hypoxia-inducible factor 1 in clinical and experimental aortic aneurysm disease. J Vasc Surg 68: 1538-1550.e2, 2018.

43. Watts ER and Walmsley SR: Inflammation and hypoxia: HIF and PHD isoform selectivity. Trends Mol Med 25: 33-46, 2019. 